Homology, Homotopy and Applications, vol.19(2), 2017, pp.111-130

\title{
TWISTED SIMPLICIAL GROUPS AND TWISTED HOMOLOGY OF CATEGORIES
}

\author{
J. Y. LI, V. V. VERSHININ AND J. WU \\ (communicated by Graham Ellis)
}

\begin{abstract}
Let $A$ be either a simplicial complex $K$ or a small category $\mathcal{C}$ with $V(A)$ as its set of vertices or objects. We define a twisted structure on $A$ with coefficients in a simplicial group $G$ as a function

$$
\delta: V(A) \longrightarrow \operatorname{End}(G), \quad v \mapsto \delta_{v},
$$

such that $\delta_{v} \circ \delta_{w}=\delta_{w} \circ \delta_{v}$ if there exists an edge in $A$ joining $v$ with $w$ or an arrow either from $v$ to $w$ or from $w$ to $v$. We give a canonical construction of twisted simplicial groups as well as twisted homology for $A$ with a given twisted structure. Also we determine the homotopy type of this simplicial group as the loop space over certain twisted smash product.
\end{abstract}

\section{Introduction}

In 1959 there appeared the works of Barratt, Gugenheim and Moore [3] and Brown [5]. In the first, the authors studied the notion of simplicial fibre-bundle. In the second, twisted tensor products were introduced and studied. Since that time a twisting of standard structures of algebraic topology appeared in various contexts and in many works. In particular, Witten [26], Rohm and Witten [23] introduced a variation $(d+H)$ of the De Rham differential $d$. The cohomology that is obtained is called twisted De Rham cohomology now. Recently some new versions of homology (cohomology) theory and combinatorial homotopy theory of graphs and similar objects were defined $[\mathbf{1}, \mathbf{2}, \mathbf{7}, \mathbf{9}-\mathbf{1 2}, \mathbf{2 4}, \mathbf{2 5}]$. The classical simplicial homology was varied by considering (co-)homology with local coefficient system for colored posets $[\mathbf{9}, \mathbf{2 5}]$ or by inserting $\delta$-factors as numerical data on vertices in the differentials of simplicial chain complexes $[\mathbf{1 0}, \mathbf{1 1}]$.

On the other hand in 1955 James $[\mathbf{1 5}]$ gave a reduced product construction $J(X)$ of a topological space $X$ with given basepoint which is the free monoid generated by $X$. For a connected CW complex $X$, the James reduced product $J(X)$ has the same homotopy type as $\Omega \Sigma X$, the loop space of the suspension of $X$. In 1984 Carlsson generalized the James construction for the case of $G$-spaces, where $G$ is a discrete group.

Received August 29, 2016; published on October 18, 2017.

2010 Mathematics Subject Classification: Primary 55U10; Secondary 18G30.

Key words and phrases: homology, simplicial group, category.

Article available at http://dx.doi.org/10.4310/HHA.2017.v19.n2.a7

Copyright (c) 2017, J. Y. Li, V. V. Vershinin and J. Wu. Permission to copy for private use granted. 
The purpose of this paper is to give a canonical twisted construction of simplicial groups as well as twisted homology for a simplicial complex and a small category with a given twisted structure. In particular, our twisted homology (twisted cohomology) is a generalization of the $\delta$-homology $(\delta$-cohomology) introduced in $[\mathbf{1 0}, \mathbf{1 1}]$ and our constructions are different from the twisted Cartesian product introduced by Barratt, Gugenheim and Moore [3], and Brown [5] and the balanced product introduced by Carlsson [6].

Categories considered in the paper are small and we omit mentioning it. All necessary definitions will be given later in the text of the paper.

Let $A$ be either a simplicial complex $K$ or a category $\mathcal{C}$. We are considering category from the geometrical point of view, using the terminology of underlying quiver, for example we are using the word "vertices" instead of objects. However, composition of morphisms and identity morphisms are involved in our constructions. Let $V(A)$ be the vertex set of $A$, and $G$ a simplicial group. We denote by $\operatorname{End}(G)$ the monoid of simplicial endomorphisms of $G$, namely $\operatorname{End}(G)$ consists of graded endomorphisms $f=\left\{f_{n}: G_{n} \rightarrow G_{n}\right\}_{n} \geqslant 0$ such that $d_{i} \circ f=f \circ d_{i}$ and $s_{i} \circ f=f \circ s_{i}$ for the face homomorphisms $d_{i}: G_{n} \rightarrow G_{n-1}$ and the degeneracy homomorphisms $s_{i}: G_{n} \rightarrow G_{n+1}$ for $0 \leqslant i \leqslant n$. A twisted structure on $A$ with coefficients in a simplicial group $G$ is a function

$$
\delta: V(A) \longrightarrow \operatorname{End}(G), \quad v \mapsto \delta_{v},
$$

satisfying the following commuting rule:

$$
\delta_{v} \circ \delta_{w}=\delta_{w} \circ \delta_{v}
$$

if there exists an edge in $A$ (if $A$ is a simplicial complex) joining $v$ with $w$ or an arrow either from $v$ to $w$ or from $w$ to $v$ (if $A$ is a category). A twisted structure on $A$ with coefficients in $G$ is called regular if $\delta_{v}: G \rightarrow G$ is a simplicial automorphism for each $v \in V(A)$.

Now let us suppose that a simplicial complex $K$ has a total order. There is a canonical associated simplicial set $S(K)$ by allowing the repeating of vertices in the definition of elements of $S(K)$. For a category $\mathcal{C}$ take $S=S(\mathcal{C})$ to be a nerve of $\mathcal{C}$. The simplicial set $S=S(A)$ has the following nature: the set $S_{n}$ of $n$-paths ( $n$-simplices) has a form of the sequences

$$
v_{0} \alpha_{1} v_{1} \cdots \alpha_{n} v_{n}
$$

with $v_{i} \in V(\mathcal{C})$ and $\alpha_{i}: v_{i-1} \rightarrow v_{i}$ a morphism of the category $\mathcal{C}$ or the sequences $\left(v_{0}, v_{1}, \ldots, v_{n}\right)$ for $v_{i} \in V(K)$ with $v_{i} \leqslant v_{j}$ for $i \leqslant j$ under a total order on $V(K)$ and $\left\{v_{0}, \ldots, v_{n}\right\}$ being a simplex in $K$. The face operation $d_{i}: S_{n} \rightarrow S_{n-1}, 0 \leqslant i \leqslant n$, is given by removing the vertex $v_{i}$ followed by the composition $\alpha_{i} \alpha_{i+1}: v_{i-1} \rightarrow v_{i+1}$, and the degeneracy operation $s_{i}: S_{n} \rightarrow S_{n+1}, 0 \leqslant i \leqslant n$, is given by doubling the vertex $v_{i}$ with inserting the identity arrow $e_{v_{i}}: v_{i} \rightarrow v_{i}$ if it is the case of category.

It follows from (1) that the functions $\delta_{v_{i}}$ and $\delta_{v_{j}}$ satisfy the commuting rule on all pairs $\left(v_{i}, v_{j}\right)$ of vertices of arbitrary $n$-path.

We construct a $\Delta$-group and a simplicial group $F_{\delta}^{G}[A]$ depending on the twisted structure $\delta$. This $\Delta$-group seems to be new. If the $\delta$-structure is given by $\delta_{v} \equiv \mathrm{id}_{G}$ for $v \in V(A)$, then $F_{\delta}^{G}[A]$ is connected with Carlsson's $J_{G}(X)$-construction [6] or Quillen's tensor product [21, Chapter II, Section 3, page 3.7] with its geometric 
realization having the homotopy type of $\Omega(B|G| \wedge|A|)$, where $|X|$ is the geometric realization of a simplicial set $X$.

As a consequence of the main result (Theorem 4.5) in this work we determine the homotopy type of $F_{\delta}^{G}[A]$ as the loop space of the $\delta$-twisted smash product $B|G| \wedge_{\delta}$ $|A|$ (see Section 4 for the definition of $\delta$-twisted smash product). Observe that the homotopy type of Carlsson's $J_{G}(X)$-construction is the loop space of the reduced Borel construction of $G$-action on $|X|\left[\mathbf{6}\right.$, Theorem 9]. The construction $F_{\delta}^{G}[A]$ gives a new type of simplicial group.

One of the points of the main result (Theorem 4.5) is to give a topological interpretation of the $\delta$-homology ( $\delta$-cohomology) introduced in $[\mathbf{1 0}, \mathbf{1 1}]$.

The results of this work can be generalized to the category of digraphs following the lines of the papers $[\mathbf{1 1}, \mathbf{1 2}]$.

The article is organized as follows. In Section 2, we give a review on the path complexes of categories. The twisted construction of the $\Delta$-groups and simplicial groups is given in Section 3 by examining the $\Delta$-identity and simplicial identities. The homotopy type of the twisted simplicial group $F_{\delta}^{G}[A]$ is studied in Section 4 , where the main result is Theorem 4.5. In Section 5, we explore the twisted homology of simplicial complexes as well as categories.

\section{Acknowledgments}

The first author is supported by NSFC (11201314, 11302136, A2014210062) of China and the Excellent Young Scientist Fund of Shijiazhuang Tiedao University. The second author is partially supported by the CNRS PICS project of cooperation with Georgia, No 237647. The last author is partially supported by the Singapore Ministry of Education research grant (AcRF Tier 1 WBS No. R-146-000-222-112) and a grant (No. 11329101) of NSFC of China.

The second author is grateful to Daniel Tanré for useful remarks.

\section{Path complexes of categories and simplicial set extension of simplicial complexes}

\subsection{Path complexes of categories}

Recall that a quiver is a quadruple $\mathcal{Q}=(V(\mathcal{Q}), E(\mathcal{Q}), s, t)$, where the set $V$ is called the set of vertices of $\mathcal{Q}$, the set $E$ is called the set of edges of $\mathcal{Q}, s$ and $t$ are two maps $s: E \rightarrow V$, giving the start or source of the edge, and $t: E \rightarrow V$, giving the target of the edge. The elements in $E(\mathcal{Q})$ we denote by arrows $\alpha: v \rightarrow w$, or $v \alpha w$.

Recall also that there is a forgetful functor from small categories to quivers

$$
\text { Cat } \rightarrow \text { Quiv. }
$$

By an $n$-path for a category $\mathcal{C}$ we mean the sequence $v_{0} \alpha_{1} \cdots v_{n-1} \alpha_{n} v_{n}$ with $n \geqslant 1$. There exists a trivial path (the identity arrow) $e_{v}: v \rightarrow v$ for each vertex $v \in V(\mathcal{C})$. Let $S_{n}(\mathcal{C})$ be the set of all $n$-paths with $n \geqslant 1$ and $S_{0}(\mathcal{C})=V(\mathcal{C})$.

The face operations in $S(\mathcal{C})$ are defined as follows: $d_{0}$ removes the initial vertex together with the first arrow and the last face, $d_{n}$ removes the last vertex together with 
the last arrowhighlight, and for $1 \leqslant i \leqslant n-1$ the operation $d_{i}$ is given by removing a vertex followed by the composition of arrows in the following sense:

$$
d_{i}\left(v_{0} \alpha_{1} v_{1} \alpha_{2} v_{2} \cdots \alpha_{n} v_{n}\right)=v_{0} \alpha_{1} v_{1} \cdots v_{i-1}\left(\alpha_{i+1} \circ \alpha_{i}\right) v_{i+1} \cdots \alpha_{n} v_{n} .
$$

The degeneracy operations

$$
s_{i}: S_{n}(\mathcal{C}) \longrightarrow S_{n+1}(\mathcal{C}),
$$

for $0 \leqslant i \leqslant n$ are defined by doubling the $i$-vertex with inserting the trivial path. Then $S(\mathcal{C})=\left\{S_{n}(\mathcal{C})\right\}_{n \geqslant 0}$ is the classifying simplicial set (or nerve) of the category $\mathcal{C}$. Choose a vertex $a_{0} \in V(\mathcal{C})$ as a basepoint. Consider the constant $n$-path $a_{0}^{n}=a_{0} e_{a_{0}} a_{0} \cdots e_{a_{0}} a_{0}$ as a basepoint for the set $S_{n}(\mathcal{C})$. Then $S(\mathcal{C})$ is a pointed simplicial set [27, Chapter 2].

\subsection{Simplicial set extension of simplicial complexes}

Let $K$ be a simplicial complex with its vertex set $V(K)$ which has a total order. Recall that the simplicial set $S(K)$ induced by $K$ is given as follows:

1. The set $S(K)_{n}$ consists of sequences $\left(v_{0}, v_{1}, \ldots, v_{n}\right)$ of vertices of $K$ such that $v_{0} \leqslant v_{1} \leqslant \cdots \leqslant v_{n}$ and $\left\{v_{0}, \ldots, v_{n}\right\}$ forms a simplex in $K$.

2. The face operation $d_{i}: S(K)_{n} \rightarrow S(K)_{n-1}, 0 \leqslant i \leqslant n$, is given by

$$
d_{i}\left(v_{0}, v_{1}, \ldots, v_{n}\right)=\left(v_{0}, v_{1}, \ldots, v_{i-1}, v_{i+1}, \ldots, v_{n}\right) .
$$

3. The degeneracy operation $s_{i}: S(K)_{n} \rightarrow S(K)_{n+1}, 0 \leqslant i \leqslant n$, is given by

$$
s_{i}\left(v_{0}, v_{1}, \ldots, v_{n}\right)=\left(v_{0}, v_{1}, \ldots, v_{i-1}, v_{i}, v_{i}, v_{i+1}, \ldots, v_{n}\right) .
$$

The geometric realization of the simplicial set $S(K)$ is homeomorphic to the polyhedron $|K|$ (see, for example, $[\mathbf{8}]$ ). We choose a basepoint in $S(K)_{n}$ by $a_{0}^{n}=\left(a_{0}, a_{0}, \ldots\right.$, $\left.a_{0}\right)$ for a vertex $a_{0} \in V(K)$ so that $S(K)$ is a pointed simplicial set.

\section{The twisted construction of $\Delta$-groups and simplicial groups}

A $\Delta$-set is a sequence of sets $X=\left\{X_{n}, n \geqslant 0\right\}$ with maps called faces

$$
d_{i}: X_{n} \rightarrow X_{n-1}, 0 \leqslant i \leqslant n,
$$

such that the following condition holds

$$
d_{i} d_{j}=d_{j} d_{i+1},
$$

for $i \geqslant j$. It is called the $\Delta$-identity $[\mathbf{2 7}]$. Roughly speaking a $\Delta$-set is a simplicial set without degeneracies.

A $\Delta$-set $G=\left\{G_{n}\right\}_{n \geqslant 0}$ is called a $\Delta$-group if each $G_{n}$ is a group, and each face $d_{i}$ is a group homomorphism [27].

We carry out the following steps for constructing a $\Delta$-group and a simplicial group depending on the twisted structure $\delta$ on $A$ :

1) Choose a vertex $a_{0} \in V(A)$ as a basepoint. Consider the constant $n$-path $a_{0}^{n}=$ $a_{0} e_{a_{0}} a_{0} \cdots e_{a_{0}} a_{0}$ as a basepoint for the set $S_{n}$. Define $F^{G}[A]_{n}$ to be the free product of the group $G_{n}$ with indices running over all non-basepoint elements in $S_{n}(A)$. More precisely, let $\left(G_{n}\right)_{x}$ be a copy of $G_{n}$ labeled by an element 
$x \in S_{n}(A)$. Then $F^{G}[A]_{n}$ is the quotient group of the free product $*_{x \in S_{n}}\left(G_{n}\right)_{x}$ subject to the relations that $\left(G_{n}\right)_{a_{0}^{n}}=\{1\}$.

2) Let $g_{x}$ denote the element $g \in G_{n}$ in its copy group $\left(G_{n}\right)_{x}$ labeled by $x$. Define a (twisted) face operation

$$
d_{i}^{\delta}: F^{G}[A]_{n} \rightarrow F^{G}[A]_{n-1}, 0 \leqslant i \leqslant n
$$

to be the (unique) group homomorphism such that $d_{i}^{\delta}$ restricted to each copy $\left(G_{n}\right)_{v_{0} \alpha_{1} v_{1} \cdots \alpha_{n} v_{n}}$ is given by the twisted formula

$$
\left.d_{i}^{\delta}\right|_{\left(G_{n}\right)_{v_{0} \alpha_{1} v_{1} \cdots \alpha_{n} v_{n}}}\left(g_{v_{0} \alpha_{1} v_{1} \cdots \alpha_{n} v_{n}}\right)=\left(\delta_{v_{i}}\left(d_{i}(g)\right)\right)_{d_{i}\left(v_{0} \alpha_{1} v_{1} \cdots \alpha_{n} v_{n}\right)} .
$$

Namely the element $d_{i} g \in\left(G_{n-1}\right)_{d_{i}\left(v_{0} \alpha_{1} v_{1} \cdots \alpha_{n} v_{n}\right)}$ is twisted by the endomorphism $\delta_{v_{i}}: G_{n-1} \rightarrow G_{n-1}$ By Proposition 3.1 below, the sequence of groups $F_{\delta}^{G, \Delta}[A]=\left\{F^{G}[A]_{n}\right\}_{n \geqslant 0}$ with the twisted face operations forms a $\Delta$-group, where the commuting rule (1) assures the $\Delta$-identity.

$3)$ Suppose that the $\delta$-structure is regular. We define a twisted degeneracy operation $s_{i}^{\delta}: F^{G}[A]_{n} \rightarrow F^{G}[A]_{n+1}, 0 \leqslant i \leqslant n$, to be the (unique) group homomorphism such that $s_{i}^{\delta}$ restricted to each copy $\left(G_{n}\right)_{v_{0} \alpha_{1} v_{1} \cdots \alpha_{n} v_{n}}$ is given by the twisted formula

$$
\left.s_{i}^{\delta}\right|_{\left(G_{n}\right)_{v_{0} \alpha_{1} v_{1} \cdots \alpha_{n} v_{n}}}\left(g_{v_{0} \alpha_{1} v_{1} \cdots \alpha_{n} v_{n}}\right)=\left(\left(\delta_{v_{i}}\right)^{-1}\left(s_{i}(g)\right)\right)_{s_{i}\left(v_{0} \alpha_{1} v_{1} \cdots \alpha_{n} v_{n}\right)} .
$$

By Proposition 3.1, the sequence of groups $F_{\delta}^{G}[A]=\left\{F^{G}[A]_{n}\right\}_{n \geqslant 0}$ with the twisted face operations $d_{i}^{\delta}$ in step 2 and the above twisted degeneracy operations $s_{i}^{\delta}$ forms a simplicial group [27, Chapter 2], where the commuting rule (1) and invertibility of $\delta_{v}$ are essential for assuring the simplicial identities.

The $\Delta$-group $F_{\delta}^{G, \Delta}[A]$ is a generalization of $\delta$-homology $[\mathbf{1 0}, \mathbf{1 1}]$ in the following sense. Let $G$ be any abelian group. Let $\delta: V(A) \rightarrow \operatorname{End}(G)$ be a function satisfying the commuting rule (1). We consider $G$ as a discrete simplicial group with $G_{n}=G$ and the faces and degeneracies given by the identity. Then we have the $\delta$-structure on $A$. By taking the abelianization of the (non-commutative) $\Delta$-group $F_{\delta}^{G, \Delta}[A]$ in step 2, we have an abelian $\Delta$-group with the twisted faces given in (3) on chains

$$
\left(F_{\delta}^{G}[A]_{n}\right)^{\mathrm{ab}}=\left(\bigoplus_{x \in S_{n}} G_{x}\right) /(G)_{a_{0}^{n}}
$$

having the differentials given by

$$
\partial_{n}\left(g_{v_{0} \alpha_{1} v_{1} \cdots \alpha_{n} v_{n}}\right)=\sum_{i=0}^{n}(-1)^{i}\left(\delta_{v_{i}}\left(d_{i}(g)\right)\right)_{d_{i}\left(v_{0} \alpha_{1} v_{1} \cdots \alpha_{n} v_{n}\right)} .
$$

In the case where $G$ is a commutative $\operatorname{ring} R$ and $\delta_{v}$ is the translation given by an element $\delta_{v} \in R$, the above formula on the differentials is exactly given by inserting the factor $\delta_{v}$ as described in $[\mathbf{1 0}, \mathbf{1 1}]$.

Proposition 3.1. Let $S$ be $S(A)$ where $A$ is either a simplicial complex $K$ or a category $\mathcal{C}$ with twisted structure in a simplicial group $G$. Then 
1. The sequence of groups $F_{\delta}^{G, \Delta}[A]=\left\{F^{G}[A]_{n}\right\}_{n \geqslant 0}$ with the twisted face operations defined by formula 3 forms a $\Delta$-group.

2. Suppose that the twisted structure $\delta$ is regular. Then the sequence of groups $F_{\delta}^{G}[A]=\left\{F^{G}[A]_{n}\right\}_{n \geqslant 0}$ with the twisted face operations $d_{i}^{\delta}$ given by formula 3 and the twisted degeneracy operations $s_{i}^{\delta}$ given by formula 4 forms a simplicial group.

Proof. The proof is given by examining the $\Delta$-identity for assertion (1), and the simplicial identities for assertion (2). Since the examination on the $\Delta$-identity for assertion (1) is part of the simplicial identities for assertion (2), we only need to prove assertion (2) with paying attention that the invertibility of $\delta_{v}$ is not required in the $\Delta$-identity for face operations.

For the basepoint $a_{0}^{n}$ in $S_{n}$, we see that each $d_{i}^{\delta}$ sends $\left(G_{n}\right)_{a_{0}^{n}}$ into $\left(G_{n-1}\right)_{a_{0}^{n-1}}$, so

$$
d_{i}^{\delta}: F_{\delta}^{G}[A]_{n} \rightarrow F_{\delta}^{G}[A]_{n-1}
$$

is a well-defined homomorphism. The same is true for

$$
s_{i}^{\delta}: F_{\delta}^{G}[A]_{n} \rightarrow F_{\delta}^{G}[A]_{n+1} .
$$

Now we show that the simplicial-identity holds for the face and degeneracy operations. For simplicity of notation, we only consider the case $S=S(K)$. For the case $S(\mathcal{C})$ of a category $\mathcal{C}$, the proof follows along the same lines, keeping in mind the following rules:

1) When a vertex $v_{i}$ is removed from the sequence $\left(v_{0}, \ldots, v_{n}\right)$, the arrow from $v_{i-1} \rightarrow v_{i+1}$ in the sequence $\left(v_{0}, \ldots, v_{i-1}, v_{i+1}, \ldots, v_{n}\right)$ is given by the composition $v_{i-1} \rightarrow v_{i} \rightarrow v_{i+1}$.

2) When a vertex $v_{i}$ is doubled from the sequence $\left(v_{0}, \ldots, v_{n}\right)$, we insert the trivial path $e_{v_{i}}: v_{i} \rightarrow v_{i}$ in the sequence

$$
\left(v_{0}, \ldots, v_{i-1}, v_{i}, v_{i}, v_{i+1}, \ldots, v_{n}\right) .
$$

Let $g_{\left(v_{0}, \ldots, v_{n}\right)} \in\left(G_{n}\right)_{\left(v_{0}, \ldots, v_{n}\right)}$, and let $i \geqslant j$ with $0 \leqslant j \leqslant n-1$ and $0 \leqslant i \leqslant n$. Then

$$
\begin{aligned}
d_{i}^{\delta} \circ d_{j}^{\delta}\left(g_{\left(v_{0}, \ldots, v_{n}\right)}\right) & =d_{i}^{\delta}\left(\delta_{v_{j}}^{n-1}\left(d_{j} g\right)_{\left(v_{0}, \ldots, v_{j-1}, v_{j+1}, \ldots, v_{n}\right)}\right) \\
& =d_{i}^{\delta}\left(d_{j}\left(\delta_{v_{j}}^{n}(g)\right)_{\left(v_{0}, \ldots, v_{j-1}, v_{j+1}, \ldots, v_{n}\right)}\right) \\
& =\left(\delta_{v_{i+1}}^{n-2}\left(d_{i}\left(d_{j}\left(\delta_{v_{j}}^{n}(g)\right)\right)\right)\right)_{\left(v_{0}, \ldots, v_{j-1}, v_{j+1}, \ldots, v_{i}, v_{i+2}, \ldots, v_{n}\right)} \\
& =\left(d_{i}\left(d_{j}\left(\delta_{v_{i+1}}^{n}\left(\delta_{v_{j}}^{n}(g)\right)\right)\right)\right)_{\left(v_{0}, \ldots, v_{j-1}, v_{j+1}, \ldots, v_{i}, v_{i+2}, \ldots, v_{n}\right)} \\
& =\left(d_{j}\left(d_{i+1}\left(\delta_{v_{j}}^{n}\left(\delta_{v_{i+1}}^{n}(g)\right)\right)\right)_{\left(v_{0}, \ldots, v_{j-1}, v_{j+1}, \ldots, v_{i}, v_{i+2}, \ldots, v_{n}\right)}\right. \\
& =\left(d_{j}\left(\delta_{v_{j}}^{n-1}\left(d_{i+1}\left(\delta_{v_{i+1}}^{n}(g)\right)\right)\right)\right)_{d_{j} d_{i+1}\left(v_{0}, \ldots, v_{n}\right)} \\
& \left.=\left(\delta_{v_{j}}^{n-2}\left(d_{j}\left(\delta_{v_{i+1}}^{n-1}\left(d_{i+1}(g)\right)\right)\right)\right)_{d_{j} d_{i+1}\left(v_{0}, \ldots, v_{n}\right)}\right) \\
& =d_{j}^{\delta} \circ d_{i+1}^{\delta}\left(g_{\left(v_{0}, \ldots, v_{n}\right)}\right)
\end{aligned}
$$

where $\delta_{v_{i+1}}^{n} \circ \delta_{v_{j}}^{n}=\delta_{v_{j}}^{n} \circ \delta_{v_{i+1}}^{n}$ because $v_{j} v_{i+1}$ forms a 1-simplex in $K$. (In the case of $S=S(\mathcal{C}), \delta_{v_{i+1}}^{n} \circ \delta_{v_{j}}^{n}=\delta_{v_{j}}^{n} \circ \delta_{v_{i+1}}^{n}$ because there is an arrow given by a composition from $v_{j}$ to $v_{i+1}$.) 
Let $i \geqslant j$, and let $g_{\left(v_{0}, \ldots, v_{n}\right)} \in\left(G_{n}\right)_{\left(v_{0}, \ldots, v_{n}\right)}$. We shorten the notation $s_{i}^{\delta}$ as $s_{i}$. Then

$$
\begin{aligned}
s_{j} s_{i}\left(g_{\left(v_{0}, \ldots, v_{n}\right)}\right) & =s_{j}\left(\left(\delta_{v_{i}}^{n+1}\right)^{-1}\left(s_{i}(g)\right)_{\left(v_{0}, \ldots, v_{i}, v_{i}, \ldots, v_{n}\right)}\right) \\
& =s_{j}\left(s_{i}\left(\left(\delta_{v_{i}}^{n}\right)^{-1}(g)\right)_{\left(v_{0}, \ldots, v_{i}, v_{i}, \ldots, v_{n}\right)}\right) \\
& =\left(\delta_{v_{j}}^{n+2}\right)^{-1}\left(s_{j} s_{i}\left(\left(\delta_{v_{i}}^{n}\right)^{-1}(g)\right)\right)_{\left(v_{0}, \ldots, v_{j}, v_{j}, \ldots, v_{i}, v_{i}, \ldots, v_{n}\right)} \\
& =\left(s_{j} s_{i}\left(\delta_{v_{j}}^{n}\right)^{-1}\left(\left(\delta_{v_{i}}^{n}\right)^{-1}(g)\right)\right)_{\left(v_{0}, \ldots, v_{j}, v_{j}, \ldots, v_{i}, v_{i}, \ldots, v_{n}\right)} \\
& =\left(s_{j} s_{i}\left(\delta_{v_{i}}^{n}\right)^{-1}\left(\left(\delta_{v_{j}}^{n}\right)^{-1}(g)\right)\right)_{\left(v_{0}, \ldots, v_{j}, v_{j}, \ldots, v_{i}, v_{i}, \ldots, v_{n}\right)} \\
& =\left(\delta_{v_{i}}^{n+2}\right)^{-1}\left(s_{i+1}\left(\left(\delta_{v_{j}}^{n+1}\right)^{-1} s_{j}(g)\right)\right)_{\left(v_{0}, \ldots, v_{j}, v_{j}, \ldots, v_{i}, v_{i}, \ldots, v_{n}\right)} \\
& =s_{i+1} s_{j}\left(g_{\left(v_{0}, \ldots, v_{n}\right)}\right) .
\end{aligned}
$$

We shorten the notation $d_{i}^{\delta}$ as $d_{i}$. Let $i<j$, and let $g_{\left(v_{0}, \ldots, v_{n}\right)} \in\left(G_{n}\right)_{\left(v_{0}, \ldots, v_{n}\right)}$. Then

$$
\begin{aligned}
d_{i} s_{j}\left(g_{\left(v_{0}, \ldots, v_{n}\right)}\right) & =d_{i}\left(\left(\delta_{v_{j}}^{n+1}\right)^{-1}\left(s_{j}(g)\right)_{\left(v_{0}, \ldots, v_{j}, v_{j}, \ldots, v_{n}\right)}\right) \\
& =d_{i}\left(\left(s_{j}\left(\delta_{v_{j}}^{n}\right)^{-1}(g)_{\left(v_{0}, \ldots, v_{j}, v_{j}, \ldots, v_{n}\right)}\right)\right. \\
& =\delta_{v_{i}}^{n}\left(d_{i}\left(s_{j}\left(\delta_{v_{j}}^{n}\right)^{-1}(g)\right)\right)_{\left(v_{0}, \ldots, v_{i-1}, v_{i+1}, \ldots, v_{j}, v_{j}, \ldots, v_{n}\right)} \\
& =\left(d_{i} s_{j} \delta_{v_{i}}^{n}\left(\delta_{v_{j}}^{n}\right)^{-1}(g)\right)_{\left(v_{0}, \ldots, v_{i-1}, v_{i+1}, \ldots, v_{j}, v_{j}, \ldots, v_{n}\right)} \\
& =\left(s_{j-1} d_{i}\left(\delta_{v_{j}}^{n}\right)^{-1}\left(\delta_{v_{i}}^{n}\right)(g)\right)_{\left(v_{0}, \ldots, v_{i-1}, v_{i+1}, \ldots, v_{j}, v_{j}, \ldots, v_{n}\right)} \\
& =\left(\left(\delta_{v_{j}}^{n}\right)^{-1}\left(s_{j-1}\left(\delta_{v_{i}}^{n-1}\left(d_{i}(g)\right)\right)\right)\right)_{\left(v_{0}, \ldots, v_{i-1}, v_{i+1}, \ldots, v_{j}, v_{j}, \ldots, v_{n}\right)} \\
& =s_{j-1} d_{i}\left(g_{\left(v_{0}, \ldots, v_{n}\right)}\right)
\end{aligned}
$$

Let $i=j$, and let $g_{\left(v_{0}, \ldots, v_{n}\right)} \in\left(G_{n}\right)_{\left(v_{0}, \ldots, v_{n}\right)}$. Then

$$
\begin{aligned}
d_{j} s_{j}\left(g_{\left(v_{0}, \ldots, v_{n}\right)}\right) & =d_{j}\left(\left(\delta_{v_{j}}^{n+1}\right)^{-1}\left(s_{j}(g)\right)_{\left(v_{0}, \ldots, v_{j}, v_{j}, \ldots, v_{n}\right)}\right) \\
& =d_{j}\left(\left(s_{j}\left(\delta_{v_{j}}^{n}\right)^{-1}(g)\right)_{\left(v_{0}, \ldots, v_{j}, v_{j}, \ldots, v_{n}\right)}\right) \\
& =\left(\delta_{v_{j}}^{n}\left(d_{j} s_{j}\left(\delta_{v_{j}}^{n}\right)^{-1}(g)\right)\right)_{\left(v_{0}, \ldots, v_{n}\right)} \\
& =\left(d_{j} s_{j}\left(\delta_{v_{j}}^{n}\left(\delta_{v_{j}}^{n}\right)^{-1}(g)\right)\right)_{\left(v_{0}, \ldots, v_{n}\right)} \\
& =g_{\left(v_{0}, \ldots, v_{n}\right)} .
\end{aligned}
$$

Let $i=j+1$, and let $g_{\left(v_{0}, \ldots, v_{n}\right)} \in\left(G_{n}\right)_{\left(v_{0}, \ldots, v_{n}\right)}$. We remind that the rule is such that we add the twisting of the vertex we are removing. When we apply $d_{j+1}$, we should add twisting $\delta_{v_{j}}$ because the second $v_{j}$ is located in the position $(j+1)$ under removing. Then we have

$$
\begin{aligned}
d_{j+1} s_{j}\left(g_{\left(v_{0}, \ldots, v_{n}\right)}\right) & =d_{j+1}\left(\left(\delta_{v_{j}}^{n+1}\right)^{-1}\left(s_{j}(g)\right)_{\left(v_{0}, \ldots, v_{j}, v_{j}, \ldots, v_{n}\right)}\right) \\
& =d_{j+1}\left(\left(s_{j}\left(\delta_{v_{j}}^{n}\right)^{-1}(g)\right)_{\left(v_{0}, \ldots, v_{j}, v_{j}, \ldots, v_{n}\right)}\right) \\
& =\delta_{v_{j}}^{n}\left(d_{j+1} s_{j}\left(\delta_{v_{j}}^{n}\right)^{-1}(g)\right)_{\left(v_{0}, \ldots, v_{n}\right)} \\
& =\left(d_{j+1} s_{j}\left(\delta_{v_{j}}^{n}\left(\delta_{v_{j}}^{n}\right)^{-1}(g)\right)\right)_{\left(v_{0}, \ldots, v_{n}\right)} \\
& =g_{\left(v_{0}, \ldots, v_{n}\right)} .
\end{aligned}
$$

Let $i>j+1$, and let $g_{\left(v_{0}, \ldots, v_{n}\right)} \in\left(G_{n}\right)_{\left(v_{0}, \ldots, v_{n}\right)}$. Then

$$
\begin{aligned}
d_{i} s_{j}\left(g_{\left(v_{0}, \ldots, v_{n}\right)}\right) & =d_{i}\left(\left(\delta_{v_{j}}^{n+1}\right)^{-1}\left(s_{j}(g)\right)_{\left(v_{0}, \ldots, v_{j}, v_{j}, \ldots, v_{n}\right)}\right) \\
& =d_{i}\left(\left(s_{j}\left(\delta_{v_{j}}^{n}\right)^{-1}(g)\right)_{\left(v_{0}, \ldots, v_{j}, v_{j}, \ldots, v_{n}\right)}\right) \\
& =\delta_{v_{i-1}}^{n}\left(d_{i}\left(s_{j}\left(\delta_{v_{j}}^{n}\right)^{-1}(g)\right)\right)_{\left(v_{0}, \ldots, v_{j}, v_{j}, \ldots, v_{i-2}, v_{i}, \ldots, v_{n}\right)} \\
& =\left(d_{i} s_{j} \delta_{v_{i-1}}^{n}\left(\delta_{v_{j}}^{n}\right)^{-1}(g)\right)_{\left(v_{0}, \ldots, v_{j}, v_{j}, \ldots, v_{i-2}, v_{i}, \ldots, v_{n}\right)} \\
& =\left(s_{j} d_{i-1}\left(\delta_{v_{j}}^{n}\right)^{-1}\left(\delta_{v_{i-1}}^{n}\right)(g)\right)_{\left(v_{0}, \ldots, v_{j}, v_{j}, \ldots, v_{i-2}, v_{i}, \ldots, v_{n}\right)} \\
& =\left(\left(\delta_{v_{j}}^{n}\right)^{-1}\left(s_{j}\left(\delta_{v_{i-1}}^{n-1}\left(d_{i-1}(g)\right)\right)\right)\right)_{\left(v_{0}, \ldots, v_{j}, v_{j}, \ldots, v_{i-2}, v_{i}, \ldots, v_{n}\right)} \\
& =s_{j} d_{i-1}\left(g_{\left(v_{0}, \ldots, v_{n}\right)}\right) .
\end{aligned}
$$




\section{Homotopy type of $F_{\delta}^{\mathcal{G}}[S]$}

\subsection{The $\delta$-twisted Cartesian products}

We generalize now the notion of twisted structure for a simplicial set, not necessarily a simplicial group $G$. Let $Y$ be a simplicial set. We denote by $\operatorname{End}(Y)$ the monoid of self-simplicial maps of $Y$. Let $A$ be a simplicial complex or a category with vertex set $V(A)$. A twisted structure on $A$ in a simplicial set $Y$ is a function

$$
\delta: V(A) \longrightarrow \operatorname{End}(Y), \quad v \mapsto \delta_{v},
$$

such that the commuting rule

$$
\delta_{v} \circ \delta_{w}=\delta_{w} \circ \delta_{v}
$$

holds if there is an edge joint $v$ and $w$ in the case of simplicial complexes and arrow between $v$ and $w$ in the case of category. A twisted structure on $A$ is called regular if $\delta_{v}: Y \rightarrow Y$ is a simplicial isomorphism for each $v \in V(A)$.

Let $S=S(A)$ be a simplicial set defined in Section 2. Given any twisted structure $\delta$ on $A$ in a simplicial set $Y$, we can carry out the following construction of $\delta$-twisted Cartesian product:

1. The sequence of sets is given by $Y_{n} \times S_{n}$ for $n \geqslant 0$.

2. The faces $d_{i}: Y_{n} \times S_{n} \rightarrow Y_{n-1} \times S_{n-1}, 0 \leqslant i \leqslant n$, are defined by the formula

$$
d_{i}\left(y, v_{0} \alpha_{1} v_{1} \cdots \alpha_{n} v_{n}\right)=\left(\delta_{v_{i}}\left(d_{i} y\right), d_{i}\left(v_{0} \alpha_{1} v_{1} \cdots \alpha_{n} v_{n}\right)\right) .
$$

3. Suppose that the twisted structure $\delta$ is regular. We define the degeneracy operation $s_{i}: Y_{n} \times S_{n} \rightarrow Y_{n+1} \times S_{n+1}, 0 \leqslant i \leqslant n$, by the formula

$$
s_{i}\left(y, v_{0} \alpha_{1} v_{1} \cdots \alpha_{n} v_{n}\right)=\left(\delta_{v_{i}}^{-1}\left(s_{i} y\right), s_{i}\left(v_{0} \alpha_{1} v_{1} \cdots \alpha_{n} v_{n}\right)\right) .
$$

Remark 4.1. The definition of our $\delta$-twisted Cartesian product is different from that of the classical twisted Cartesian product in simplicial theory $[\mathbf{3}, \mathbf{8}]$.

Proposition 4.2. Let $S=S(A)$ for $A$ with a twisted structure $\delta$ in a simplicial set $Y$. Then

1. The sequence of sets $Y \times_{\delta, \Delta} S=\left\{Y_{n} \times S_{n}\right\}_{n \geqslant 0}$ with the face operations defined by (8) forms a $\Delta$-set.

2. Suppose that the twisted structure $\delta$ is regular. Then the sequence of sets $Y \times_{\delta}$ $S=\left\{Y_{n} \times S_{n}\right\}_{n \geqslant 0}$ with the face operations defined in (8) and the degeneracy operations defined by (9) forms a simplicial set.

Proof. The assertions follow along the lines in the proof of Proposition 3.1 for examining the $\Delta$-identity and the simplicial identities.

The first assertion of the above proposition is sufficient for having homology (cohomology) with twisted coefficients. Let $A$ have a twisted structure $\delta$ in a simplicial set $Y$. Let $G$ be an abelian group. The twisted homology (twisted cohomology) of $A$ with coefficients in $G$ is defined by

$$
H_{n}^{\text {twist }}(A ; G)=H_{n}\left(G\left(Y \times_{\delta, \Delta} S(A)\right)\right), H_{\text {twist }}^{n}(A ; G)=H^{n}\left(G\left(Y \times_{\delta, \Delta} S(A)\right)\right),
$$

for $n \geqslant 0$, where $G(X)$ for a simplicial set $X$ is given by $G(X)=\oplus_{x \in X}(G)_{x}$ with $(G)_{x}$ a copy of $G$ labeled by $x$. A consequence of (1) of Proposition (4.2) is the following corollary. 
Corollary 4.3. Let $A$ have a twisted structure $\delta$ in a simplicial set $Y$. Then the twisted homology $H_{*}^{\text {twist }}(A ; G)$ (twisted cohomology $H_{\text {twist }}^{*}(A ; G)$ ) is the homology (cohomology) of the chain complex $C_{*}^{\text {twist }}(A)$ with coefficients in $G$, where

$$
C_{n}^{\text {twist }}(A)=\mathbb{Z}\left(Y_{n}\right) \otimes \mathbb{Z}\left(S(A)_{n}\right),
$$

with the differential $\partial_{n}^{\text {twist }}: C_{n}^{\text {twist }}(A) \rightarrow C_{n-1}^{\text {twist }}(A)$ given by the formula

$$
\partial_{n}\left(y \otimes v_{0} \alpha_{1} v_{1} \cdots \alpha_{n} v_{n}\right)=\sum_{i=0}^{n}(-1)^{i} \delta_{v_{i}}\left(d_{i} y\right) \otimes d_{i}\left(v_{0} \alpha_{1} v_{1} \cdots \alpha_{n} v_{n}\right),
$$

for $n \geqslant 0$.

Connections between cartesian product and tensor product is usual in Algebraic Topology, they start with the Eilenberg-Zilber theorem.

From the second assertion of Proposition 4.2, there is a connection between $\delta$ twisted Cartesian products and simplicial fibre bundles which is described as follows. Let $S=S(A)$ have a regular twisted structure $\delta$ in a simplicial set $Y$. Observe that the coordinate projection

$$
p: Y \times_{\delta} S \rightarrow S, \quad\left(g, v_{0} \alpha_{1} v_{1} \cdots \alpha_{n} v_{n}\right) \mapsto v_{0} \alpha_{1} v_{1} \cdots \alpha_{n} v_{n}
$$

is a simplicial map. Recall from [8, page 155] that a simplicial map $p: E \rightarrow B$ is called a simplicial fibre bundle with fibre $F$ if for each $b \in B_{n}$, there is a commutative diagram

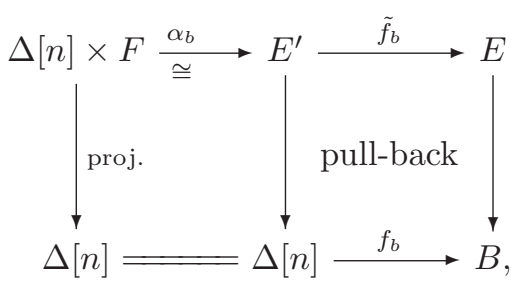

where $\Delta[n]$ is the standard simplicial $n$-simplex, $f_{b}: \Delta[n] \rightarrow B$ is the representing map of $b \in B_{n}$ and $\alpha_{b}$ is a simplicial isomorphism.

Theorem 4.4. Let $S=S(A)$ have a regular twisted structure $\delta$ in a simplicial set $Y$. Then the coordinate projection map

$$
p: Y \times_{\delta} S \rightarrow S
$$

is a simplicial fibre bundle with fibre $Y$.

Proof. Let $\sigma=(0,1, \ldots, n) \in \Delta[n]_{n}$ be the standard non-degenerate $n$-simplex. For each $b=v_{0} \alpha_{1} v_{1} \cdots \alpha_{n} v_{n} \in S_{n}$, we have $f_{b}(\sigma)=b$ by definition. By taking iterated faces, we have $f_{b}(i)=v_{i}$. The twisted structure on $A$ induces a function

$$
\delta: V(\Delta[n])=\{(0),(1), \ldots,(n)\} \longrightarrow \operatorname{End}(Y)
$$

given by

$$
\delta_{(i)}=\delta_{v_{i}}
$$

for $0 \leqslant i \leqslant n$. For $0 \leqslant i<j \leqslant n$, since there is an edge between $v_{i}$ and $v_{j}$ or an 
arrow $v_{i} \rightarrow v_{j}$, we have

$$
\delta_{(i)} \circ \delta_{(j)}=\delta_{(j)} \circ \delta_{(i)},
$$

by the commuting rule $\delta_{v_{i}} \circ \delta_{v_{j}}=\delta_{v_{j}} \circ \delta_{v_{i}}$ and so a twisted structure on $\Delta[n]$ in $Y$. From the definition of $\delta$-twisted Cartesian product, we have the simplicial map

$$
\left(\text { id, } f_{b}\right): Y \times_{\delta} \Delta[n] \longrightarrow E=Y \times_{\delta} S
$$

inducing a fibrewise simplicial isomorphism

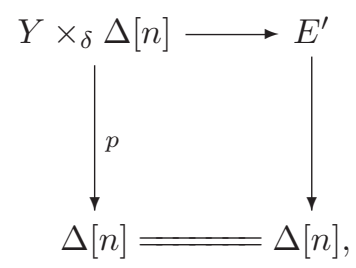

where $E^{\prime}$ is given by the pull-back in diagram (10) with $E=Y \times{ }_{\delta} S$ and $B=S$. Now we show that there is a fibrewise simplicial isomorphism

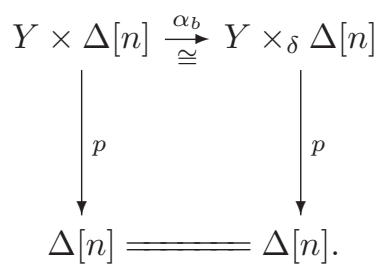

The simplicial isomorphism $\alpha_{b}$ is constructed by untwisting the twisted faces and degeneracies. More precisely, observe that, each element $w$ in $\Delta[n]_{q}$ can be uniquely expressed as a monotone sequence

$$
w=(\overbrace{j_{1}(w), \ldots, j_{1}(w)}^{l_{1}(w)}, \ldots, \overbrace{j_{t_{w}}(w), \ldots, j_{t_{w}}(w)}^{l_{t_{w}}(w)}),
$$

with $l_{i}(w) \geqslant 1$ for $1 \leqslant i \leqslant t, 0 \leqslant j_{1}(w)<j_{2}(w)<\cdots<j_{t_{w}}(w) \leqslant n$ and $\sum_{i=1}^{t_{w}} l_{i}(w)=$ $q+1$. Let

$$
\left\{i_{1}(w), \ldots, i_{s_{w}}(w)\right\}=\{0,1, \ldots, n\} \backslash\left\{j_{1}(w), j_{2}(w), \ldots, j_{t_{w}}(w)\right\},
$$

with $i_{1}(w)<i_{2}(w)<\cdots<i_{s_{w}}(w)$ be the set of missing vertices in $w$. Define the function

$$
\alpha_{b}: Y_{q} \times \Delta[n]_{q} \longrightarrow Y_{q} \times \Delta[n]_{q}
$$

by setting

$$
\alpha_{b}(y, w)=\left(\delta_{\left(j_{t_{w}}(w)\right)}^{-l_{t_{w}(w)}+1} \cdots \delta_{\left(j_{1}(w)\right)}^{-l_{1}(w)+1} \delta_{\left(i_{1}(w)\right)} \cdots \delta_{\left(i_{s_{w}}(w)\right)}(y), w\right) .
$$

Clearly $\alpha_{b}: Y_{q} \times \Delta[n]_{q} \longrightarrow Y_{q} \times \Delta[n]_{q}$ is bijective for $q \geqslant 0$. It suffices to show that $\alpha_{b}$ is a simplicial map. We shorten the notation $l_{i}(w)$ as $l_{i}$, and similarly for other 
functions on $w$. Note that, for $0 \leqslant i \leqslant q$,

$$
\begin{aligned}
d_{i}^{\delta}\left(\alpha_{b}(y, w)\right) & =d_{i}^{\delta}\left(\delta_{\left(j_{t}\right)}^{-l_{t}+1} \cdots \delta_{\left(j_{1}\right)}^{-l_{1}+1} \delta_{\left(i_{1}\right)} \cdots \delta_{\left(i_{s}\right)}(y), w\right) \\
& =\left(\delta_{\left(i^{\prime}\right)}\left(d_{i} \delta_{\left(j_{t}\right)}^{-l_{t}+1} \cdots \delta_{\left(j_{1}\right)}^{-l_{1}+1} \delta_{\left(i_{1}\right)} \cdots \delta_{\left(i_{s}\right)}(y)\right), d_{i}(w)\right) \\
& =\left(\delta_{\left(i^{\prime}\right)} \delta_{\left(j_{t}\right)}^{-l_{t}+1} \cdots \delta_{\left(j_{1}\right)}^{-l_{1}+1} \delta_{\left(i_{1}\right)} \cdots \delta_{\left(i_{s}\right)}\left(d_{i}(y)\right), d_{i}(w)\right),
\end{aligned}
$$

where $\left(i^{\prime}\right)$ is the $i$-th vertex of the $q$-simplex $w$. Let $k$ be the minimal integer such that

$$
l_{1}+\cdots+l_{k}-1 \geqslant i
$$

Then $i^{\prime}=j_{k}$ and

$$
d_{i}^{\delta}\left(\alpha_{b}(y, w)\right)=\left(\delta_{\left(j_{t}\right)}^{-l_{t}+1} \cdots \delta_{j_{k}}^{-l_{k}+2} \cdots \delta_{\left(j_{1}\right)}^{-l_{1}+1} \delta_{\left(i_{1}\right)} \cdots \delta_{\left(i_{s}\right)}\left(d_{i}(y)\right), d_{i}(w)\right) .
$$

Note that

$$
d_{i} w=(\overbrace{j_{1}(w), \ldots, j_{1}(w)}^{l_{1}(w)}, \ldots, \overbrace{j_{k}(w), \ldots, j_{k}(w)}^{l_{k}(w)-1}, \ldots, \overbrace{j_{t_{w}}(w), \ldots, j_{t_{w}}(w)}^{l_{t_{w}}(w)}) .
$$

From the definition of $\alpha_{b}$, we have

$$
\alpha_{b}\left(d_{i}(y), d_{i}(w)\right)=d_{i}^{\delta}\left(\alpha_{b}(y, w)\right) .
$$

Consider the degeneracy operation $s_{i}, 0 \leqslant i \leqslant q$. We have

$$
s_{i} w=(\overbrace{j_{1}(w), \ldots, j_{1}(w)}^{l_{1}(w)}, \ldots, \overbrace{j_{k}(w), \ldots, j_{k}(w)}^{l_{k}(w)+1}, \ldots, \overbrace{j_{t_{w}}(w), \ldots, j_{t_{w}}(w)}^{l_{t_{w}}(w)}) .
$$

Then

$$
\begin{aligned}
s_{i}^{\delta}\left(\alpha_{b}(y, w)\right) & =s_{i}^{\delta}\left(\delta_{\left(j_{t}\right)}^{-l_{t}+1} \cdots \delta_{\left(j_{1}\right)}^{-l_{1}+1} \delta_{\left(i_{1}\right)} \cdots \delta_{\left(i_{s}\right)}(y), w\right) \\
& =\left(\delta_{\left(j_{k}\right)}^{-1}\left(s_{i} \delta_{\left(j_{t}\right)}^{-l_{t}+1} \cdots \delta_{\left(j_{1}+1\right.}^{-l_{1}+1} \delta_{\left(i_{1}\right)} \cdots \delta_{\left(i_{s}\right)}(y)\right), s_{i}(w)\right) \\
& =\left(\delta_{\left(j_{k}\right)}^{-1} \delta_{\left(j_{t}\right)}^{-l_{t}+1} \cdots \delta_{\left(j_{1}\right)}^{-l_{1}+1} \delta_{\left(i_{1}\right)} \cdots \delta_{\left(i_{s}\right)}\left(s_{i}(y)\right), s_{i}(w)\right) \\
& =\left(\delta_{\left(j_{t}\right)}^{-l_{t}+1} \cdots \delta_{\left(j_{k}\right)}^{-l_{k}} \cdots \delta_{\left(j_{1}\right)}^{-l_{1}+1} \delta_{\left(i_{1}\right)} \cdots \delta_{\left(i_{s}\right)}\left(s_{i}(y)\right), s_{i}(w)\right) \\
& =\alpha_{b}\left(s_{i}(y), s_{i}(w)\right) .
\end{aligned}
$$

\subsection{The $\delta$-twisted smash products}

Now we consider the pointed constructions. Let $Y$ be a pointed simplicial set with the base-point $*=\left\{s_{0}^{n} *\right\}_{n \geqslant 0}$. A reduced twisted structure on $A$ in $Y$ is a function

$$
\delta: V(K) \longrightarrow \operatorname{End}_{*}(Y), \quad v \mapsto \delta_{v},
$$

such that the commuting rule $(7)$ holds, where $\operatorname{End}_{*}(Y)$ is the monoid of base-pointpreserving self-simplicial maps of $Y$. A reduced twisted structure on $A$ is called regular if $\delta_{v}: Y \rightarrow Y$ is a simplicial isomorphism for each $v \in V(A)$.

Suppose that $S=S(A)$ has regular reduced twisted structure $\delta$ in a pointed simplicial set $Y$. Since $\delta_{v}(*)=*$ for $v \in V(A)$ there is a canonical inclusion

$$
* \times S=* \times_{\delta} S \longleftrightarrow Y \times_{\delta} S \text {. }
$$

Let $a_{0}$ be a vertex of $A$ treated as the base-point with the induced $\delta$-structure by the 
restriction. Then there is a canonical simplicial inclusion

$$
Y \times{ }_{\delta} a_{0} \longrightarrow Y \times{ }_{\delta} S \text {. }
$$

The $\delta$-twisted smash product $Y \wedge_{\delta} S$ is then defined as the simplicial quotient set

$$
Y \wedge_{\delta} S:=\left(Y \times_{\delta} S\right) /\left(\left(Y \times_{\delta} a_{0}\right) \cup\left(* \times_{\delta} S\right)\right) .
$$

\subsection{The classifying spaces of the twisted simplicial groups}

Recall that there is a functor $\bar{W}$ from simplicial groups to simplicial sets, which plays the role of the classifying space functor in simplicial theory. The construction of the functor $\bar{W}$ is briefly reviewed using the terminology of categorical digraphs as follows.

Let $G$ be a group (without assuming simplicial structure). Let $\mathcal{G}$ be the category with a single vertex $a$ and a collection of arrows labeled by the elements $g \in G$, where the identity $1=e_{a}: a \rightarrow a$ is considered as the trivial path of the vertex $a$. Then $\mathcal{G}$ is a categorical digraph with the composition operation induced by the multiplication of the group $G$. Let $W(G)=S(\mathcal{G})$ defined in Section 2 .

Now let $G=\left\{G_{n}\right\}_{n \geqslant 0}$ be a simplicial group. The face homomorphisms $d_{i}: G_{n} \rightarrow$ $G_{n-1}$ and the degeneracy homomorphisms $s_{i}: G_{n} \rightarrow G_{n+1}$ induce simplicial face maps $W\left(d_{i}\right): W\left(G_{n}\right) \rightarrow W\left(G_{n-1}\right)$ and simplicial degeneracy maps $W\left(s_{i}\right): W\left(G_{n}\right) \rightarrow$ $W\left(G_{n+1}\right)$ for $0 \leqslant i \leqslant n$ so that $\left\{W\left(G_{n}\right)\right\}_{n \geqslant 0}$ is a bi-simplicial set. We recall that a bi-simplicial set is a simplicial object in the category of simplicial sets [14]. The simplicial set $\bar{W}(G)$ is then defined as the diagonal simplicial set of the bi-simplicial set $W\left(G_{*}\right)_{*}$.

Theorem 4.5. Let $S=S(A)$ have a regular twisted structure in a simplicial group $G$. Then there is a natural simplicial map

$$
\theta: \bar{W}(G) \wedge_{\delta} S \longrightarrow \bar{W}\left(F_{\delta}^{G}[S]\right),
$$

which is a homotopy equivalence after geometric realization, where the twisted structure of $A$ in the simplicial set $\bar{W}(G)$ is induced from its twisted structure in $G$ through the functor $\bar{W}$.

Proof. Let $v$ be a vertex of $A$. By applying the functor $\bar{W}$ to the simplicial isomorphism $\delta_{v}: G \rightarrow G$, we have a sequence of isomorphisms of pointed simplicial sets

$$
\bar{W}\left(\delta_{v}^{n}\right): \bar{W}\left(G_{n}\right) \longrightarrow \bar{W}\left(G_{n}\right)
$$

and the commutative diagram

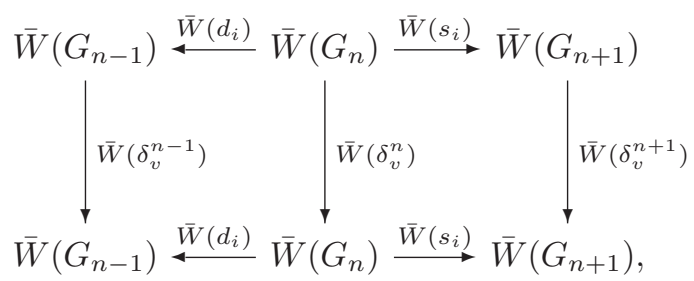

for $0 \leqslant i \leqslant n$ and $v \in V(A)$.

Recall from the definition that the group $\left(F_{\delta}^{G}[S]\right)_{n}=F^{G}[S]_{n}$ is the quotient group of the free product $*_{x \in S_{n}}\left(G_{n}\right)_{x}$ subject to the relations $\left(G_{n}\right)_{a_{0}^{n}}=\{1\}$. For $x \in S_{n}$, 
the inclusion $\left(G_{n}\right)_{x} \longleftrightarrow F^{G}[S]_{n}$ induces a simplicial map

$$
j_{x}: \bar{W}\left(\left(G_{n}\right)_{x}\right) \longrightarrow \bar{W}\left(\left(F_{\delta}^{G}[S]\right)_{n}\right)
$$

and so a simplicial map

$$
\tilde{\theta}_{n}: \bar{W}\left(G_{n}\right) \times S_{n} \longrightarrow \bar{W}\left(\left(F_{\delta}^{G}[S]\right)_{n}\right) \quad(y, x) \mapsto j_{x}(y),
$$

where we consider $S_{n}$ as a discrete simplicial set with $\left(S_{n}\right)_{q}=S_{n}$ for $q \geqslant 0$ and faces and degeneracies being given by the identity map. Since $\left(G_{n}\right)_{a_{0}^{n}}=\{1\}$, we have

$$
\tilde{\theta}_{n}\left(\bar{W}\left(G_{n}\right) \times\left\{a_{0}^{n}\right\}\right)=* .
$$

Since $j_{x}(*)=*$ for any $x \in S_{n}$, we have

$$
\tilde{\theta}_{n}\left(* \times S_{n}\right)=*
$$

and so $\tilde{\theta}$ factors through the smash product. Let

$$
\theta_{n}: \bar{W}\left(G_{n}\right) \wedge S_{n} \longrightarrow \bar{W}\left(\left(F_{\delta}^{G}[S]\right)_{n}\right)
$$

be the resulting simplicial map. Now we let $n$ be varied. Let

$$
\begin{gathered}
x=v_{0} \alpha_{1} v_{1} \cdots \alpha_{n} v_{n} \in S_{n}=\left(S_{n}\right)_{q} \text { and } \\
y=a g_{1} a g_{2} a \cdots g_{q} a \in \bar{W}\left(G_{n}\right)_{q},
\end{gathered}
$$

with $g_{1}, \ldots, g_{q} \in G_{n}$. Then

$$
\theta_{n}(y \wedge x)=a\left(g_{1}\right)_{x} a\left(g_{2}\right)_{x} a \cdots\left(g_{q}\right)_{x} a .
$$

Under the twisted faces in the simplicial group $F_{\delta}^{G}[S]$, we have

$$
\begin{aligned}
\bar{W}\left(d_{i}^{\delta}\right)\left(\theta_{n}(y \wedge x)\right) & =\bar{W}\left(d_{i}^{\delta}\right)\left(a\left(g_{1}\right)_{x} a\left(g_{2}\right)_{x} a \cdots\left(g_{q}\right)_{x} a\right. \\
& =a\left(d_{i}^{\delta}\left(\left(g_{1}\right)_{x}\right)\right) a\left(d_{i}^{\delta}\left(\left(g_{2}\right)_{x}\right)\right) a \cdots\left(d_{i}^{\delta}\left(\left(g_{q}\right)_{x}\right)\right) a \\
& =a\left(\left(\delta_{v_{i}}^{n-1}\left(d_{i}\left(g_{1}\right)\right)\right)_{d_{i} x}\right) a \cdots\left(\left(\delta_{v_{i}}^{n-1}\left(d_{i}\left(g_{q}\right)\right)\right)_{d_{i} x}\right) a \\
& =\theta_{n-1}\left(\left(\bar{W}\left(\delta_{v_{i}}^{n-1}\right)\left(d_{i}(y)\right)\right) \wedge d_{i}(x)\right),
\end{aligned}
$$

for $0 \leqslant i \leqslant n$. Similarly, under the twisted faces in the simplicial group $F_{\delta}^{G}[S]$, we have

$$
\bar{W}\left(s_{i}^{\delta}\left(\theta_{n}(y \wedge x)\right)=\theta_{n+1}\left(\left(\bar{W}\left(\left(\delta_{v_{i}}^{n+1}\right)^{-1}\right)\left(s_{i}(y)\right)\right) \wedge s_{i}(x)\right)\right) .
$$

We define a $\delta$-twisted structure on the sequence of simplicial sets

$$
\left\{\bar{W}\left(G_{n}\right) \wedge S_{n}\right\}_{n \geqslant 0},
$$

by the formulae

$$
\begin{gathered}
d_{i}^{\delta}(y \wedge x)=\left(\bar{W}\left(\delta_{v_{i}}^{n-1}\right)\left(d_{i}(y)\right)\right) \wedge d_{i}(x), \\
\left.s_{i}^{\delta}(y \wedge x)=\left(\bar{W}\left(\left(\delta_{v_{i}}^{n+1}\right)^{-1}\right)\left(s_{i}(y)\right)\right) \wedge s_{i}(x)\right),
\end{gathered}
$$

for $0 \leqslant i \leqslant n, x=v_{0} \alpha_{1} v_{1} \cdots \alpha_{n} v_{n} \in S_{n}=\left(S_{n}\right)_{q}$ and $y \in \bar{W}\left(G_{n}\right)_{q}$. Since each $\theta_{n}$ is injective and $\bar{W}\left(\left(F_{\delta}^{G}[S]\right)_{*}\right)_{*}$ is a bi-simplicial set, the simplicial identities hold for $d_{i}^{\delta}$ and $s_{i}^{\delta}$ in $T_{*, *}=\left\{\bar{W}\left(G_{n}\right) \wedge S_{n}\right\}_{n \geqslant 0}$ so that $T_{*, *}$ is a bi-simplicial set with

$$
\theta_{*}: T_{*, *} \longrightarrow \bar{W}\left(\left(F_{\delta}^{G}[S]\right)_{*}\right)_{*}
$$

being a morphism of bi-simplicial sets. 
Recall that, by the Whitehead Theorem [18, Proposition 4.3], the canonical inclusion

$$
\bar{W}\left(G^{\prime}\right) \vee \bar{W}\left(G^{\prime \prime}\right) \rightarrow \bar{W}\left(G^{\prime} * G^{\prime \prime}\right)
$$

is a homotopy equivalence after geometric realization for any simplicial groups $G^{\prime}$ and $G^{\prime \prime}$. It follows that

$$
\theta_{n}: \bar{W}\left(G_{n}\right) \wedge S_{n} \longrightarrow \bar{W}\left(\left(F_{\delta}^{G}[S]\right)_{n}\right)
$$

is a homotopy equivalence after geometric realization for $n \geqslant 0$. According to [4], the geometric realization

$$
\left|\theta_{*}\right|:\left|T_{*, *}\right| \longrightarrow\left|\bar{W}\left(\left(F_{\delta}^{G}[S]\right)_{*}\right)_{*}\right|
$$

is a homotopy equivalence. Moreover, by [4], the geometric realization of the diagonal simplicial set associated to a bi-simplicial set $X_{*, *}$ is homotopy equivalent to $\left|X_{*, *}\right|$. From the definition of the functor $\bar{W}$, the simplicial set $\bar{W}\left(F_{\delta}^{G}[S]\right)$ is given by the diagonal simplicial set of the bi-simplicial set $\bar{W}\left(\left(F_{\delta}^{G}[S]\right)_{*}\right)_{*}$. By the definition of $\delta$-twisted smash product, it is evident that the diagonal simplicial set of $T_{*, *}$ is $\bar{W}(G) \wedge_{\delta} S$.

Let $|X|$ denote the geometric realization of a simplicial set $X$. By Theorem 4.5, we have the following.

Corollary 4.6. Let $S=S(A)$ have a regular twisted structure in a simplicial group $G$. Then the topological group $\left|F_{\delta}^{G}[S]\right|$ has the homotopy type of $\Omega\left|\bar{W}(G) \wedge_{\delta} S\right|$.

If a twisted structure is trivial then the realization of our construction gives the same homotopy type as the Carlsson' $J_{G}(X)$-construction.

Corollary 4.7. Let $S=S(A)$ with twisted structure in a simplicial group $G$ given by $\delta_{v} \equiv \operatorname{id}_{G}$ for $v \in V(A)$. Then the topological group $\left|F_{\delta}^{G}[S]\right|$ has the homotopy type of $\Omega|B(G) \wedge S|$. If in this case $G=\mathbb{Z}$, the we have Milnor's $F[K]$ construction.

Proof. The assertion follows from Corollary 4.6 and the definition of $\delta$-twisted smash product.

Example. Let $G$ be a simplicial group $\mathbb{Z}$ concentrated on dimension 0 . Let $S$ be the simplicial circle $S^{1}$ given as the boundary of a 2 -simplex. Let $\delta$ be involution for every vertex. Then the $\delta$-twisted Cartesian product $\bar{W}(G) \times{ }_{\delta} S^{1}$ is the Klein bottle. From (11), there is a cofibre sequence

$$
S^{1} \vee S^{1} \longrightarrow \bar{W}(G) \times_{\delta} S^{1} \longrightarrow \bar{W}(G) \wedge_{\delta} S^{1}
$$

and so $\bar{W}(G) \wedge_{\delta} S^{1} \simeq S^{2}$. Thus $F_{\delta}^{G}\left[S^{1}\right]$ is a simplicial group model for $\Omega S^{2}$.

\section{Twisted homology of simplicial complexes and categories}

\subsection{Remarks on twisted homology}

In this subsection, we give some remarks on the twisted $\Delta$-groups $F_{\delta}^{G, \Delta}[S]$, where $S=S(A)$ with a twisted structure $\delta$ in a simplicial group $G$. The construction $F_{\delta}^{G, \Delta}[S]$ seems to be general, which only requires the commuting rule (1), and so one has the 
homology groups from the chain complexes given by $\mathbb{Z}\left(F_{\delta}^{G, \Delta}[S]\right)$ and $\left(F_{\delta}^{G, \Delta}[S]\right)^{\text {ab }}$ (as well as $\mathbb{Z}\left(\bar{W}(G) \times_{\delta, \Delta} S\right)$ in Section 4.1) for any twisted structure. On the other hand, the $\Delta$-groups $F_{\delta}^{G, \Delta}[S]$ could be very wild in general. For instance, if $\delta_{v}$ is the trivial endomorphism of $G$ for any vertex $v$, all twisted face operations in $F_{\delta}^{G, \Delta}[S]$ become trivial, which concludes that the differentials in $\mathbb{Z}\left(F_{\delta}^{G, \Delta}[S]\right),\left(F_{\delta}^{G, \Delta}[S]\right)^{\text {ab }}$ and $\mathbb{Z}\left(\bar{W}(G) \times_{\delta, \Delta} S\right)$ are zero maps and so their homology is given by the chains themselves. This indicates that the homology groups from the chain complexes given by $\mathbb{Z}\left(F_{\delta}^{G, \Delta}[S]\right),\left(F_{\delta}^{G, \Delta}[S]\right)^{\text {ab }}$ and $\mathbb{Z}\left(\bar{W}(G) \times_{\delta, \Delta} S\right)$ may not be homotopy invariants for general twisted structures $\delta$. If the twisted structure is regular, the homology groups from the chain complexes given by $\mathbb{Z}\left(F_{\delta}^{G, \Delta}[S]\right)$ and $\mathbb{Z}\left(\bar{W}(G) \times_{\delta, \Delta} S\right)$ coincide with the homology groups from the chain complexes given by the simplicial abelian groups $\mathbb{Z}\left(F_{\delta}^{G}[S]\right)$ and $\mathbb{Z}\left(\bar{W}(G) \times{ }_{\delta} S\right)$ by $[\mathbf{8}$, Section 5$]$ and so Theorems 4.4 and 4.5 assure that these homology groups only depend on the homotopy type of the corresponding fibre bundles.

Some simplicial techniques can be used for understanding the twisted $\Delta$-groups $F_{\delta}^{G, \Delta}[S]$.

Proposition 5.1. Let $S=S(K)$ for a simplicial complex $K$ with a twisted structure in a simplicial group $G$. Let $K_{1}$ and $K_{2}$ be simplicial sub-complexes of $K$ such that $K_{1} \cup K_{2}=K$ and $K_{1} \cap K_{2} \neq \emptyset$. Let $\delta_{K_{i}}$ be the restriction of the twisted structure $\delta_{K}$ on $K_{i}$. We choose the basepoint in $K_{1} \cap K_{2}$. Then

1. The $\Delta$-group

$$
F_{\delta}^{G, \Delta}[S(K)]=F_{\delta}^{G, \Delta}\left[S\left(K_{1}\right)\right] *_{F_{\delta}^{G, \Delta}}\left[S\left(K_{1} \cap K_{2}\right)\right] F_{\delta}^{G, \Delta}\left[S\left(K_{2}\right)\right]
$$

is the free product with amalgamation.

2. Suppose that $G$ is a simplicial abelian group. Then there is a short exact sequence of the chain complexes

$F_{\delta}^{G, \Delta}\left[S\left(K_{1} \cap K_{2}\right)\right]^{\mathrm{ab}} \longrightarrow F_{\delta}^{G, \Delta}\left[S\left(K_{1}\right)\right]^{\mathrm{ab}} \oplus F_{\delta}^{G, \Delta}\left[S\left(K_{1}\right)\right]^{\mathrm{ab}} \longrightarrow F_{\delta}^{G, \Delta}[S(K)]^{a b}$.

Proof. Assertion (2) follows from assertion (1) by taking abelianization. For proving assertion (1), we check that $S(K)=S\left(K_{1}\right) \cup_{S\left(K_{1} \cap K_{2}\right)} S\left(K_{2}\right)$. By the definition, the elements in $S(K)_{n}$ are given by $\left(v_{0}, \ldots, v_{n}\right)$ with $v_{0} \leqslant v_{1} \leqslant \cdots \leqslant v_{n}$ such that $\left\{v_{0}, \ldots, v_{n}\right\}$ form a simplex in $K$. Since $K=K_{1} \cup K_{2}$, the simplex $\left\{v_{0}, \ldots, v_{n}\right\}$ is either in $K_{1}$ or $K_{2}$. This shows that $S(K)=S\left(K_{1}\right) \cup_{S\left(K_{1} \cap K_{2}\right)} S\left(K_{2}\right)$. Assertion (1) then follows from our construction.

The second assertion in the above proposition assures that the Mayer-Vietoris sequence can be applied in the homology theory given by $H_{*}\left(F_{\delta}^{G, \Delta}[S(K)]^{a b}\right)$ for twisted simplicial complexes. Similar results hold for the case $S(\mathcal{C})$ for category $\mathcal{C}$ with twisted structure.

Now let us consider the special case of the twisted $\Delta$-groups of the cones of simplicial complexes and categories. For a simplicial complex $K$, the cone $C K=a * K$ is defined in the usual way. For a category $\mathcal{C}$, the cone $C \mathcal{C}=a * \mathcal{C}$ is the category obtained by adding an initial vertex $a$ with assigning a unique arrow $e_{a, v}: a \rightarrow v$ for each $v \in V(\mathcal{C})$ and the identical arrow id $a: a \rightarrow a$. Suppose that $\delta: V(K) \rightarrow \operatorname{End}(G)$ or $V(\mathcal{C}) \rightarrow \operatorname{End}(G)$ is a twisted structure in a simplicial group $G$. A twisted structure $\delta_{C K}\left(\right.$ or $\left.\delta_{C \mathcal{C}}\right)$ is called a regular extension of the twisted structure $\delta_{K}$ (or $\delta_{\mathcal{C}}$ ) if 
1. $\left.\delta_{C K}\right|_{V(K)}=\delta_{K}\left(\right.$ or $\left.\left.\delta_{C \mathcal{C}}\right|_{V(\mathcal{C})}=\delta_{\mathcal{C}}\right)$ and

2. $\delta(a) \in \operatorname{Aut}(G)$.

We recall that, since there is an edge or arrow between $a$ and any other vertex, the commuting rule (1) of the $\delta$-structure forces that $\delta_{a} \circ \delta_{v}=\delta_{v} \circ \delta_{a}$ for any vertex $v$.

Recall also that any group $G$ can be considered as a discrete simplicial group with $G_{n}=G$ and faces and degeneracies being the identity map.

Proposition 5.2. Let $S=S(a * A)$ where $A$ is a simplicial complex $K$ or a category $\mathcal{C}$ with a twisted structure given as a regular extension of a twisted structure on $A$ in a group $G$. Then the following chain complexes

$$
\mathbb{Z}\left(F_{\delta}^{G, \Delta}[S]\right), \quad F_{\delta}^{G, \Delta}[S]^{\text {ab }}
$$

are contractible.

Proof. Let

$$
\Phi:\left(F_{\delta}^{G, \Delta}[S]\right)_{n}=F^{G}[S]_{n} \longrightarrow\left(F_{\delta}^{G, \Delta}[S]\right)_{n+1}=F^{G}[S]_{n+1}
$$

be the (unique) group homomorphism such that

$$
\left.\Phi\right|_{\left(G_{n}\right)_{v_{0} \alpha_{1} v_{1} \cdots \alpha_{n} v_{n}}}\left(g_{v_{0} \alpha_{1} v_{1} \cdots \alpha_{n} v_{n}}\right)=\left(\delta_{a}\right)^{-1}(g)_{a e_{a, v_{0}} v_{0} \alpha_{1} v_{1} \cdots \alpha_{n} v_{n}} .
$$

Let us compute $d_{i}^{\delta} \circ \Phi$. We shorten the notation $d_{i}^{\delta}$ as $d_{i}$.

$$
\begin{aligned}
d_{0} \Phi\left(g_{v_{0} \alpha_{1} v_{1} \cdots \alpha_{n} v_{n}}\right) & =d_{0}\left(\left(\delta_{a}\right)^{-1}(g)_{a e_{a, v_{0}} v_{0} \alpha_{1} v_{1} \cdots \alpha_{n} v_{n}}\right) \\
& =\left(\delta_{a}\left(d_{0}\left(\left(\delta_{a}\right)^{-1}(g)\right)\right)\right)_{v_{0} \alpha_{1} v_{1} \cdots \alpha_{n} v_{n}} \\
& =\left(\delta_{a}\left(\left(\delta_{a}\right)^{-1}(g)\right)\right)_{v_{0} \alpha_{1} v_{1} \cdots \alpha_{n} v_{n}} \text { because } G \text { is discrete } \\
& =g_{v_{0} \alpha_{1} v_{1} \cdots \alpha_{n} v_{n} .}
\end{aligned}
$$

Thus

$$
d_{0} \Phi=\mathrm{id}
$$

Now let $i>0$. Then

$$
\begin{aligned}
d_{i} \Phi\left(g_{\left.v_{0} \alpha_{1} v_{1} \cdots \alpha_{n} v_{n}\right)}\right) & d_{i}\left(\left(\delta_{a}\right)^{-1}(g)_{a e_{a, v_{0}} v_{0} \alpha_{1} v_{1} \cdots \alpha_{n} v_{n}}\right) \\
& =\left(\delta_{v_{i-1}}\left(d_{i}\left(\delta_{a}\right)^{-1}(g)\right)\right)_{a e_{a, v_{0}} v_{0} \alpha_{1} v_{1} \cdots v_{i-2} \alpha_{i-1} \alpha_{i} v_{i} \cdots \alpha_{n} v_{n}} \\
& =\left(\left(\delta_{a}\right)^{-1} \delta_{v_{i-1}} g\right)_{a e_{a, v_{0}} v_{0} \alpha_{1} v_{1} \cdots v_{i-2} \alpha_{i-1} \alpha_{i} v_{i} \cdots \alpha_{n} v_{n}} \\
& \text { because } G \text { is discrete } \\
= & \Phi d_{i-1}\left(g_{v_{0} \alpha_{1} v_{1} \cdots \alpha_{n} v_{n}}\right) .
\end{aligned}
$$

Thus we have the following identity:

$$
d_{i} \Phi=\Phi d_{i-1} .
$$

It follows that, in the chain complex $\mathbb{Z}\left(F_{\delta}^{G, \Delta}[S]\right)$,

$$
\begin{aligned}
\partial_{n+1} \circ \Phi & =\sum_{i=0}^{n+1}(-1)^{i} d_{i} \Phi \\
& =\mathrm{id}-\sum_{i=1}^{n+1}(-1)^{i-1} \Phi d_{i-1} \\
& =\mathrm{id}-\Phi \circ \partial_{n} .
\end{aligned}
$$

and so $\mathbb{Z}\left(F_{\delta}^{G, \Delta}[S]\right)$ is contractible. By taking the abelianization, the homomorphisms $\Phi^{\mathrm{ab}}$ defines a null homotopy for the chain complex $F^{G, \Delta}[S]^{\mathrm{ab}}$. 


\subsection{An application to twisted homology}

In this subsection, we consider the abelianization $F_{\delta}^{G}[S]^{\text {ab }}$ of the simplicial group $F_{\delta}^{G}[S]$, where $S=S(A)$ for $A$ a simplicial complex $K$ or a category $\mathcal{C}$ with a regular twisted structure in an abelian group $G$. The chain complex of $F_{\delta}^{G}[S]^{\text {ab }}$ is specifically given in (5) and (6). A natural question is as follows:

Question 5.3. Does there exist a topological space whose ordinary homology gives the homology of the chain complex $F_{\delta}^{G}[S]^{\text {ab }}$ ?

As an application of Theorems 4.5 and 4.4, we affirmatively answer the above question in some special case. Let $G=\mathbb{Z}^{\oplus k}$ be a free abelian group of rank $k$. Recall that a regular twisted structure of $A$ with coefficients in $G=\mathbb{Z}^{k}$ is a function

$$
\delta: V(A) \longrightarrow \operatorname{Aut}(G)=\mathrm{GL}_{k}(\mathbb{Z})
$$

satisfying the commuting rule (1). Let $F_{k}$ be a free group of rank $k$, and let $q: \operatorname{Aut}\left(F_{k}\right) \rightarrow \mathrm{GL}_{k}(\mathbb{Z})$ be the canonical homomorphism by sending an automorphism of $F_{k}$ to the corresponding automorphism of $\mathbb{Z}^{k}=F_{k}^{\mathrm{ab}}$. We say that a twisted structure $\delta$ of $A$ with coefficients in $G=\mathbb{Z}^{\oplus k}$ is normal if $\delta$ is regular and there exists a regular twisted structure $\tilde{\delta}$ of $A$ with coefficients in the free group $F_{k}$ such that the following diagram commutes

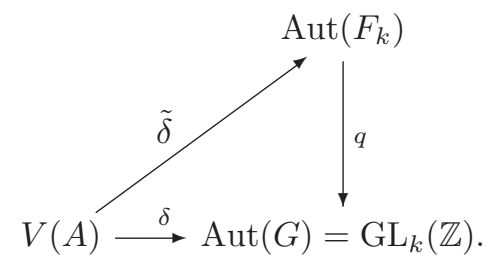

In such a case, we call $\tilde{\delta}$ a lifting of the twisted structure $\delta$ in the free group $F_{k}$.

Lemma 5.4. Let $\phi: G \rightarrow \Gamma$ be a simplicial homomorphism between simplicial groups such that $G$ and $\Gamma$ are free groups in each dimension. Suppose that $\phi$ is a homotopy equivalence. Then $\phi^{\mathrm{ab}}: G^{\mathrm{ab}} \rightarrow \Gamma^{\mathrm{ab}}$.

Proof. For a simplicial group $G$, consider the bi-simplicial set bar construction $\bar{W}_{*, *}(G)=\left\{\bar{W}\left(G_{n}\right)\right\}_{n \geqslant 0}$ with $\Delta \bar{W}_{*, *}(G)=\bar{W}(G)$. The simplicial homomorphism $\phi$ induces a bi-simplicial map $\bar{W}_{*, *}(\phi): \bar{W}_{*, *}(G) \rightarrow \bar{W}_{*, *}(\Gamma)$ with a homotopy equivalence

$$
\Delta \bar{W}_{*, *}(\phi): \Delta \bar{W}_{*, *}(G) \longrightarrow \Delta \bar{W}_{*, *}(\Gamma)
$$

by assumption. Thus

$$
\mathbb{Z}\left[\Delta \bar{W}_{*, *}(\phi)\right]: \Delta \mathbb{Z}\left[\bar{W}_{*, *}(G)\right] \longrightarrow \Delta \mathbb{Z}\left[\bar{W}_{*, *}(\Gamma)\right]
$$

is a homotopy equivalence. By Quillen [20], there is a spectral sequence with $E^{2}$ terms given by $\pi_{*}\left(\left\{\pi_{*}\left(\mathbb{Z}\left[\bar{W}\left(G_{n}\right)\right]\right)\right\}_{n \geqslant 0}\right)$ converging to $\pi_{*}\left(\Delta \mathbb{Z}\left[\bar{W}_{*, *}(G)\right]\right)$. Since each $G_{n}$ is a free group, $\pi_{k}\left(\mathbb{Z}\left[\bar{W}\left(G_{n}\right)\right]\right)=0$ for $k \neq 1$ and $\pi_{1}\left(\mathbb{Z}\left[\bar{W}\left(G_{n}\right)\right]\right) \cong G_{n}^{\text {ab }}$ with a simplicial isomorphism $G^{\mathrm{ab}} \rightarrow\left\{\pi_{1}\left(\mathbb{Z}\left[\bar{W}\left(G_{n}\right)\right]\right)\right\}_{n \geqslant 0}$. The homotopy equivalence property of $\Delta \bar{W}_{*, *}(\phi)$ implies that $\phi^{\text {ab }}$ is a homotopy equivalence.

For a reduced simplicial set $X$, let $G(X)$ denote Kan's $G$-construction [16], which is a simplicial free group whose geometric realization is homotopy equivalent to $\Omega|X|$. 
Let $Y$ be a pointed simplicial set with a basepoint $*$. Let $\mathbb{Z}[Y]=\mathbb{Z}(Y) / \mathbb{Z}(*)$ be the free abelian simplicial group generated by $Y$ modulo the basepoint $*$. Recall $[\mathbf{8}]$ that $\pi_{n}(\mathbb{Z}[Y]) \cong \tilde{H}_{n}(Y ; \mathbb{Z})$ for $n \geqslant 0$.

Theorem 5.5. Let $S=S(A)$ have a normal twisted structure $\delta$ in a free abelian group $G=\mathbb{Z}^{k}$. Let $\tilde{\delta}$ be a lifting of $\delta$ in $F_{k}$. Then there is an isomorphism

$$
H_{n}\left(F_{\delta}^{G}[S]^{\mathrm{ab}}\right) \cong \tilde{H}_{n+1}\left(\left|\bar{W}\left(F_{k}\right) \wedge_{\tilde{\delta}} S\right| ; \mathbb{Z}\right),
$$

for $n \geqslant 0$.

Proof. Let $\theta: \bar{W}\left(F_{k}\right) \wedge_{\tilde{\delta}} S \longrightarrow \bar{W} F_{\delta}^{F_{k}}[S]$ be the simplicial map in 4.5. By [17, Theorem 11.1], the simplicial map $\theta$ induces a simplicial homomorphism $\phi: G\left(\bar{W}\left(F_{k}\right) \wedge_{\tilde{\delta}}\right.$ $S) \longrightarrow F_{\tilde{\delta}}^{F_{k}}[S]$. Moreover, $\phi$ is a homotopy equivalence because $\theta$ is a homotopy equivalence after geometric realization. Since $\tilde{\delta}$ is a lifting of $\delta$, we have $F_{\tilde{\delta}}^{F_{k}}[S]^{\text {ab }}=$ $F_{\delta}^{G}[S]^{\mathrm{ab}}$. By Lemma 5.4,

$$
\phi^{\mathrm{ab}}: G\left(\bar{W}\left(F_{k}\right) \wedge_{\tilde{\delta}} S\right)^{\mathrm{ab}} \longrightarrow F_{\tilde{\delta}}^{F_{k}}[S]^{\mathrm{ab}}=F_{\delta}^{G}[S]^{\mathrm{ab}}
$$

is a homotopy equivalence. It follows that

$$
\phi_{*}^{\mathrm{ab}}: \pi_{*}\left(G\left(\bar{W}\left(F_{k}\right) \wedge_{\tilde{\delta}} S\right)^{\mathrm{ab}}\right) \longrightarrow \pi_{*}\left(F_{\delta}^{G}[S]^{\mathrm{ab}}\right) .
$$

By [8, Formula (10.1a), p.186], we have

$$
\pi_{*}\left(G\left(\bar{W}\left(F_{k}\right) \wedge_{\tilde{\delta}} S\right)^{\mathrm{ab}}\right) \cong \tilde{H}_{*+1}\left(W\left(F_{k}\right) \wedge_{\tilde{\delta}} S\right) .
$$

From $\left[\mathbf{8}\right.$, Section 5], the homology of the chain complex $F_{\delta}^{G}[S]^{\text {ab }}$ coincides with the homotopy groups of the simplicial abelian groups $F_{\delta}^{G}[S]^{\text {ab }}$. The proof is finished.

Remark 5.6. By Theorem 4.4, the coordinate projection map $\bar{W}\left(F_{k}\right) \times_{\tilde{\delta}} S \rightarrow S$ is a simplicial fibre bundle with fibre $\bar{W}\left(F_{k}\right)$. By Quillen's theorem [20], the geometric realization $\left|\bar{W}\left(F_{k}\right) \times_{\tilde{\delta}} S\right| \rightarrow|S|$ is a Serre fibration with fibre $\left|\bar{W}\left(F_{k}\right)\right|$. Since $F_{k}$ is a free group of rank $k,\left|\bar{W}\left(F_{k}\right)\right|$ is homotopy equivalent to $\mathbb{C} \backslash Q_{k}$, where $Q_{k}$ is a finite subset of $\mathbb{C}$ of cardinal $k$. In other words, the space $\left|\bar{W}\left(F_{k}\right) \times_{\tilde{\delta}} S\right|$ may be viewed up to homotopy as a quantization of $|S|$ in the sense of a punctured complex line fibration over $|S|$.

\section{References}

[1] H. Barcelo and R. Laubenbacher, Perspectives on $A$-homotopy theory and its applications. Discrete Math. 298 (2002), 39-61.

[2] H. Barcelo, X. Kramer, R. Laubenbacher, and C. Weaver, Foundations of a connectivity theory for simplicial complexes. Adv. Appl. Math. 26 (2001), 97-128.

[3] M.G. Barratt, V.K.A.M. Gugenheim, and J.C. Moore, On semisimplicial fibre-bundles. Amer. J. Math. 81 (1959), 639-657.

[4] A.K. Bousfield and E.M. Friedlander, Homotopy theory of $\Gamma$-spaces, spectra, and bisimplicial sets. Geometric Applications of Homotopy Theory (Proc. Conf., Evanston, Ill., 1977), II, pp. 80-130. Lecture Notes in Math., vol. 658, Springer, Berlin, 1978. 
[5] E.H. Brown, Jr, Twisted tensor products, I. Ann. of Math. 69 (1959), 223-246.

[6] G. Carlsson, A simplicial group construction for balanced products. Topology 23 (1984), 85-89.

[7] A. Cavicchioli and M. Meschiari, A homology theory for colored graphs. Discrete Math. 137 (1995), 99-136.

[8] E.B. Curtis, Simplicial homotopy theory. Adv. Math. 6 (1971), 107-209.

[9] B. Everitt and P. Turner, Homology of coloured posets: A generalisation of Khovanov's cube construction. J. Algebra 322 (2009), 429-448.

[10] A. Grigor'yan, Yu. Muranov, and S.-T. Yau, Graphs associated with simplicial complexes. Homology Homotopy Appl. 16 (2014), 295-311.

[11] A. Grigor'yan, Yu. Muranov, and S.-T. Yau, On a cohomology of digraphs and Hochschild cohomology. J. Homotopy Relat. Struct. 11 (2016), no. 2, 209-230.

[12] A. Grigor'yan, Y. Lin, Yu. Muranov, and S.-T. Yau, Homologies of path complexes and digraphs, preprint arXiv:1207.2834.

[13] A. Grigor'yan, Y. Lin, Yu. Muranov, and S.-T. Yau, Path complexes and their homologies, University of Bielefeld Preprint Number 13055.

[14] P.G. Goerss and J.F. Jardine, Simplicial Homotopy Theory. Birkhäuser Verlag, Basel, 2009. xvi+510 pp.

[15] I.M. James, Reduced product spaces. Ann. of Math. (2) 62 (1955), 170197.

[16] D.M. Kan, A combinatorial definition of homotopy groups. Ann. of Math. (2) 67 (1958), 282-312.

[17] D.M. Kan, On homotopy theory and c.s.s. groups. Ann. of Math. (2) 68 (1958), 38-53.

[18] D.M. Kan and W.P. Thurston, Every connected space has the homology of a $K(\pi, 1)$. Topology 15 (1976), 253-258.

[19] J.W. Milnor, On the construction FK. In: J.F. Adams, Algebraic Topology. Student's Guide. Cambridge University Press, 1972.

[20] D.G. Quillen. Spectral sequences of a double semi-simplicial group. Topology 5 (1966), 155-157.

[21] D.G. Quillen, Homotopical Algebra. Lecture Notes in Math., vol. 43. Springer-Verlag, Berlin, New York, 1967. iv+156 pp.

[22] D.G. Quillen, The geometric realization of a Kan fibration is a Serre fibration. Proc. Amer. Math. Soc. 19 (1968), 1499-1500.

[23] R. Rohm, and E. Witten, The antisymmetric tensor field in superstring theory. Ann. Phys. 170 (1986), no. 2, 454-489.

[24] M.E. Talbi and D. Benayat, Homology theory of graphs. Mediterr. J. Math. 11 (2014), 813-828.

[25] P. Turner and E. Wagner, The homology of digraphs as a generalization of Hochschild homology. J. Algebra Appl. 11 (2012), 1250031 (13 pages). 
[26] E. Witten, Supersymmetry and Morse theory. J. Differential Geom. 17 (1982), 661-692.

[27] J. Wu, Simplicial objects and homotopy groups. Braids, pp. 31-181. Lect. Notes Ser. Inst. Math. Sci. Natl. Univ. Singap., vol. 19. World Sci. Publ., Hackensack, NJ, 2010.

J. Y. Li yanjinglee@163.com

Mathematics Department, Shijiazhuang Tiedao University, Shijiazhuang 050000, China

V.V. Vershinin vladimir.verchinine@univ-montp2.fr, versh@math.nsc.ru

Département des Sciences Mathématiques, Université de Montpellier, Place Eugène Bataillon, Montpellier cedex 5, 34095, France

Sobolev Institute of Mathematics, Novosibirsk 630090, Russia

J. Wu matwuj@nus.edu.sg

Department of Mathematics, National University of Singapore, 2 Science Drive 2, Singapore 117542 